\title{
GWAS of five gynecologic diseases and cross-trait analysis in Japanese
}

\author{
Tatsuo Masuda ${ }^{1,2} \cdot$ Siew-Kee Low $\mathbb{D}^{3,4}$ - Masato Akiyama ${ }^{3,5} \cdot$ Makoto Hirata $^{6}$ - Yutaka Ueda ${ }^{2}$ Koichi Matsuda ${ }^{7}$ \\ Tadashi Kimura $^{2}$ - Yoshinori Murakami ${ }^{8} \cdot$ Michiaki Kubo $^{9} \cdot$ Yoichiro Kamatani $\mathbb{D}^{3,10} \cdot$ Yukinori Okada $\mathbb{D}^{1,11}$
}

Received: 24 January 2019 / Accepted: 12 August 2019 / Published online: 5 September 2019

(c) The Author(s) 2019. This article is published with open access

\begin{abstract}
We performed genome-wide association studies of five gynecologic diseases using data of 46,837 subjects (5236 uterine fibroid, 645 endometriosis, 647 ovarian cancer (OC), 909 uterine endometrial cancer (UEC), and 538 uterine cervical cancer (UCC) cases allowing overlaps, and 39,556 shared female controls) from Biobank Japan Project. We used the populationspecific imputation reference panel $(n=3541)$, yielding 7,645,193 imputed variants. Analyses performed under logistic model, linear mixed model, and model incorporating correlations identified nine significant associations with three gynecologic diseases including four novel findings (rs79219469:C $>\mathrm{T}$, LINC02183, $P=3.3 \times 10^{-8}$ and rs567534295:C $>\mathrm{T}$, $B R C A 1, P=3.1 \times 10^{-8}$ with OC, rs 150806792:C $>$ T, $I N S-I G F 2, P=4.9 \times 10^{-8}$ and rs $140991990: \mathrm{A}>\mathrm{G}, S O X 9, P=3.3 \times$ $10^{-8}$ with UCC). Random-effect meta-analysis of the five GWASs correcting for the overlapping subjects suggested one novel shared risk locus (rs937380553:A $>\mathrm{G}, L O C 730100, P=2.0 \times 10^{-8}$ ). Reverse regression analysis identified three additional novel associations (rs73494486:C $>\mathrm{T}, G A B B R 2, P=4.8 \times 10^{-8}$, rs $145152209: \mathrm{A}>\mathrm{G}, S H 3 G L 3 / B N C 1, P=3.3 \times$ $10^{-8}$, and rs 147427629:G > A, LOC107985484, $P=3.8 \times 10^{-8}$ ). Estimated heritability ranged from 0.026 for $\mathrm{OC}$ to 0.220 for endometriosis. Genetic correlations were relatively strong between OC and UEC, endometriosis and OC, and uterine fibroid and OC $\left(r_{g}>0.79\right)$ compared with relatively weak correlations between UCC and the other four $\left(r_{g}=-0.08 \sim 0.25\right)$. We successfully identified genetic associations with gynecologic diseases in the Japanese population. Shared genetic effects among multiple related diseases may help understanding the pathophysiology.
\end{abstract}

Supplementary information The online version of this article (https:// doi.org/10.1038/s41431-019-0495-1) contains supplementary material, which is available to authorized users.

Yukinori Okada

yokada@sg.med.osaka-u.ac.jp

1 Department of Statistical Genetics, Osaka University Graduate School of Medicine, Suita 565-0871, Japan

2 Department of Obstetrics and Gynecology, Osaka University Graduate School of Medicine, Osaka 565-0871, Japan

3 Laboratory for Statistical Analysis, RIKEN Center for Integrative Medical Sciences, Yokohama 230-0045, Japan

4 Cancer Precision Medicine Center, Japanese Foundation for Cancer Research, Tokyo 135-8550, Japan

5 Department of Ophthalmology, Graduate School of Medical Sciences, Kyushu University, Fukuoka, Fukuoka 812-8582, Japan

6 Laboratory of Genome Technology, Institute of Medical Science, the University of Tokyo, Tokyo 108-8639, Japan

\section{Introduction}

Uterine fibroma (UF), endometriosis, ovarian cancer (OC), uterine endometrial cancer (UEC), and uterine cervical cancer (UCC) are all common proliferative diseases arising

7 Department of Computational Biology and Medical Sciences, Graduate school of Frontier Sciences, The University of Tokyo, Tokyo 108-8639, Japan

8 Division of Molecular Pathology, the Institute of Medical Sciences, the University of Tokyo, Tokyo 108-8639, Japan

9 RIKEN Center for Integrative Medical Sciences, Yokohama 2300045, Japan

10 Kyoto-McGill International Collaborative School in Genomic Medicine, Graduate School of Medicine, Kyoto University, Kyoto 606-8507, Japan

11 Laboratory of Statistical Immunology, Immunology Frontier Research Center (WPI-IFReC), Osaka University, Suita 565-0871, Japan 
from gynecologic organs. They are heterogeneous diseases with diverse range of proliferative and infiltrative properties. Clinical and epidemiological studies suggest that these diseases are mutually associated or often occur as comorbidity [1]. Studies of shared background risk, namely genetics, would offer understanding of the causes of these diseases, along with identifying targets to be treated.

For the past several years, genetic studies of gynecologic diseases have revealed only a limited number of significant associations [2-10]. Of note, the common risk genes wellknown from pedigree studies, such as BRCA1 and BRCA2 [11], have not been reported as either ovarian or breast cancer-susceptibility genes in the context of genome-wide association study (GWAS) [7, 12]. This is largely because risk variants found in pedigree studies are usually rare among general population, which is unlikely detected in GWAS. So we focused on variants common among general population, including low frequency ones, to assess if these risk genes impose risks to general population, not just to specific families. The majority of the associations found in GWAS have small effect and polygenic nature. Detection of genetic associations in such cases depends on large sample size and ingenious analytical strategies. Genetic studies in the field of gynecology, including those reported from Biobank Japan Project (BBJ) [2, 4, 13], have been performed under the common logistic regression model. In this paper, to facilitate the detection of association signals and generalize the results, we conducted association studies under the liner mixed model (BOLT-LMM), with the largest GWAS data of Japanese population to date from BBJ. Advantages in adopting mixed models include that (i) they could account for both population stratification and cryptic relatedness, (ii) they could avoid confounding factors and provide robust association results, and (iii) they achieve increased statistical power for identifying genetic associations [14]. Large computational burden, a major limitation in mixed model methods especially when sample size is large, is overcome in BOLT-LMM [14]. In order to further enhance detection of novel loci, we also tried multiple association analytic approaches, where correlations between GWAS estimates among multiple related diseases are incorporated using MTAG [15]. Although MTAG utilizes summary level data and comparison between analyses based on row genotype data and summary level data might not be straightforward, the MTAG results were comparable to those from analyses under the common logistic model (mach2dat) and linear mixed model (BOLT-LMM) in a disease-specific manner. Since MTAG utilizes bivariate linkage disequilibrium (LD) score regression, where linear regression with liability threshold model is assumed and regression $z$-scores are assumed to follow standard normal distribution, which is different from the linear mixed model and non-normal distribution of regression z-scores assumed in BOLT-LMM, we utilized the results of mach2dat for applying MTAG. Also, we applied the reverse regression model using SCOPA [16], which utilizes raw genotype data, and produces estimates based on the best combination of phenotypes fitted to the model to obtain the maximized log-likelihood. We considered that SCOPA results could be comparable to those of joint analysis of all cases versus shared controls and random effect GWAS meta-analysis of different diseases in a multiple-disease-combined manner.

Clinically and epidemiologically, some of the gynecologic disorders harbor shared risk factors, such as age at menarche or menopause, and body mass index (BMI) [17-19]. These risk factors are at least partially influenced genetically and might be under the shared or pleiotropic effects of the genome [20]. To our knowledge, limited studies have investigated the shared genetic effects on gynecologic diseases, and they specifically look into relationship between two of the gynecologic diseases [21, 22], relationship among histologic subtypes [23], or include gynecologic diseases as a part of multi-disease/trait study [24]. Only one of these studies analyzed the genetic correlation between specific pair of diseases under the linear mixed model [21]. To increase our understanding of shared genetic determinants influencing gynecologic diseases, here we report genetic correlations among the five gynecologic diseases in Japanese population using the linear mixed model approach.

\section{Subjects and methods}

\section{Subjects}

In total, 7315 cases with one of the clinically diagnosed five gynecologic diseases, including UC, endometriosis, OC, UEC, and UCC including cervical intraepithelial neoplasias, and 39,829 shared female controls without gynecologic diseases were enrolled from BBJ [13]. All the subjects provided written informed consent as approved by the ethical committee of RIKEN Yokohama Institute and the Institute of Medical Science, the University of Tokyo. This study was approved by the ethical committee of Osaka University Graduate School of Medicine. Females affected with nongynecologic malignancies and/or diseases thought to be strongly associated with the major histocompatibility complex (MHC) region were excluded from the control group. Related subjects were excluded in advance to avoid possible confounding. Carrier status of known risk genes such as BRCA1 and BRCA2, histopathological subtypes, and disease severity such as tumor sizes and clinical stages were not considered. Principal component analysis (PCA) was performed using EIGENSOFT (v6.1.4) Data manipulation was performed using PLINK software (v1.90b3.3). 


\section{Genotype imputation}

Genotypes of the samples were obtained using either of the following genotyping arrays: (i) the Illumina HumanOmniExpressExome BeadChip or (ii) a combination of the Illumina HumanOmniExpress and HumanExome BeadChips. Genotype data is deposited on the Japanese Genotypephenotype Archive affiliated to the DNA Data Bank of Japan, via National Bioscience Database Center, Japan. The data are accessible on hum0014 at https://ddbj.nig.ac.jp/jga/ viewer/view/study/JGAS00000000114. For quality control (QC) of genotypes, we excluded variants meeting any of the following criteria: (i) call rate $<99 \%$, (ii) $P$-value for departure from Hardy-Weinberg equilibrium (HWE) $<1.0 \times 10^{-6}$, and (iii) number of heterozygotes less than five.

After we proceeded through these QC steps, we used Eagle (v2.3) for haplotype phasing without an external reference panel. We conducted whole-genome imputation using Minimac3 (v1.0.11) and the population-specific reference panel including multi-ethnic 2504 samples in 1000 Genomes Project (1KGP) phase 3v5a and deep whole genome sequencing of 1037 Japanese [25, 26]. Variants with minor allele frequency (MAF) $\geq 1 \%$ in both case and control subjects, and imputation info $r^{2} \geq 0.7$ were selected for the following analyses.

\section{Association analyses}

Associations of the variants with each disease were separately evaluated (i) under the logistic regression model assuming additive effects of the allele dosages using mach2dat (v1.0.24), (ii) under the linear mixed model using BOLT(-LMM) (v2.2) [14], and (iii) by incorporating correlations between GWAS summary statistics using MTAG (v1.0.7) [15] with mach2dat results. Age, squared age, BMI, and the top 20 principal components (PCs) were included as covariates. PCs were calculated from 175,574 genotyped variants using EIGENSOFT (v6.1.4) under the same QC and pruning after excluding PCA outliers as described below in Heritability and genetic correlation section.

Joint analysis of all cases versus shared controls was executed under the logistic regression model using mach2dat, and was compared with random-effect metaanalysis of mach2dat results of each disease using RE2C (v1.04) [27] to correct for the overlapping samples and increase the power for detection. The same set of analyses was also conducted under the linear mixed model using BOLT-LMM and compared with the corresponding mach2dat results. We also applied the reverse regression model to the imputed genotypes and covariates-adjusted phenotypes using SCOPA (v1.0.14) [16]. The SCOPA results were compared with those from above analyses. Since $\mathrm{RE}^{2} \mathrm{C}^{*} P$-value, which is conditioned for the overlapping samples but is not conditioned for the fixed effect, was used for plotting, genome-wide significance threshold was defined as $P=5.0 \times 10^{-8}$ for all analyses [28]. Metasoft (v2.0.1) was applied to calculate metaanalysis heterogeneity index $I^{2}$.

\section{Candidate gene and functional annotation}

For gynecologic risk loci outside of the MHC region, candidate genes were prioritized by FUMA [29]. We used the default settings except for the followings; we set the $\mathrm{LD} \mathrm{r}^{2}$ threshold to 0.5 to define LD structure of lead SNPs, reference panel population to $1 \mathrm{KGP}$ phase $3 \mathrm{EAS}$, and minimum allele frequency to 0.004 . When there are no "mapped genes" then we listed the nearest coding or noncoding genes. Since genetic architecture within the MHC region is complex [30], it is displayed as "the MHC region" instead of the gene names. Functional annotations of the identified variants were also obtained by applying GARFIELD [31] and searching through HaploReg v4.1 (see URLs).

\section{Heritability and genetic correlation}

Heritability and genetic correlation were estimated using GCTA-GREML and phenotype-correlation-genotypecorrelation (PCGC-s) (v1.0.0) [32], respectively, with the same genotyped variant matrix, which passed the QC criteria as follows; we excluded samples with call rate $<$ $99 \%$, variants with call rate $<99 \%$, variants with $\mathrm{MAF}<$ $5 \%$, variants with HWE $P$-value $<1.0 \times 10^{-6}$, variants in the MHC with flanking region spanning from 24 to $34 \mathrm{Mb}$ of chromosome 6 , then pruned variants at $\mathrm{LD} r^{2}$ cutoff of 0.50 . Disease prevalence was defined as described elsewhere [1, 33, 34]. Age, squared age, BMI, and 20 PCs were incorporated as covariates. BOLT-REML failed to converge in analyzing heritability, and GCTA-GREML failed to converge in analyzing genetic correlation, therefore we adopted PCGC-s that would definitely yield the results through Haseman-Elston regression model. PCGC-s does not produce standard errors or $p$-values for the estimates.

\section{Results}

\section{Subjects}

Numbers of the subjects eligible for each GWAS were as follows; 5236 for UF, 645 for endometriosis, 647 for OC, 909 for UEC, and 538 for UCC cases where those who have multiple diseases were allowed to enroll in each corresponding GWAS, and 39,556 shared female controls. 
Table 1 Characteristics of the genome-wide association studies of five gynecologic diseases

\begin{tabular}{llllll}
\hline Diseases & No. cases & No. controls ${ }^{\mathrm{a}}$ & Disease prevalence $^{\mathrm{b}}$ & $h_{g}^{2}{ }^{\mathrm{c}}$ & $h^{2}{ }_{S N P}^{\mathrm{d}}(\mathrm{SE})$ \\
\hline Uterine fibroid & 5236 & 39556 & $1.89 \times 10^{-1}$ & 0.26 & $1.70 \times 10^{-1}\left(2.57 \times 10^{-2}\right)$ \\
Endometriosis & 645 & 39556 & $6.94 \times 10^{-2}$ & $0.47-0.51$ & $2.20 \times 10^{-1}\left(1.30 \times 10^{-1}\right)$ \\
Ovarian cancer & 647 & 39556 & $1.22 \times 10^{-2}$ & 0.40 & $2.60 \times 10^{-2}\left(7.95 \times 10^{-2}\right)$ \\
Uterine endometrial cancer & 909 & 39556 & $1.64 \times 10^{-2}$ & 0.52 & $1.26 \times 10^{-1}\left(6.19 \times 10^{-2}\right)$ \\
Uterine cervical cancer & 538 & 39556 & $1.28 \times 10^{-2}$ & $0.11-0.34$ & $1.17 \times 10^{-1}\left(9.75 \times 10^{-2}\right)$ \\
\hline
\end{tabular}

The numbers are rounded to three significant digits for disease prevalence and $h^{2} S N P$

${ }^{\text {a }}$ Shared female controls among the five GWAS

${ }^{\mathrm{b}}$ Disease prevalence among Japanese population

${ }^{c}$ Heritability previously estimated from twin, family or population-based case-control studies

${ }^{\mathrm{d}}$ Heritability estimated from the GWAS data adjusted for disease prevalence

Characteristics of the samples included in the GWASs of the five gynecologic diseases are shown in Table 1.

\section{Genotype imputation}

Whole-genome imputation yielded $61,608,817$ variants in total. Of these variants, 7,645,193 passed the quality control as described above (MAF $\geqq 1 \%$ in both cases and controls, and imputation info $r^{2} \geqq 0.70$ ).

\section{Association analyses for single disease}

The results of single-disease association studies are shown in Fig. 1 and Table 2. The lambda values are compared in Supplementary Table 1. All variants are based on hg19. Previously reported variants are summarized in Supplementary Tables 2-6, and suggestive associations are summarized in Supplementary Tables 7-11. Functional annotations of the identified risk variants obtained by searching through HaploReg v4.1 are summarized in Supplementary Table 12. Annotations obtained by GARFIELD are shown in Supplementary Figs. 1-8. For UF, we identified four loci (rs7412010, chr1:g.22436446 G > C at 1p36, CDC42/WNT4, $\quad P=1.2 \times 10^{-12} ; \quad$ rs12415148, $\quad$ chr10: g.105680586 T $>\mathrm{C}$ at $10 \mathrm{q} 24$, STN1 (OBFC1), $P=3.5 \times$ $10^{-10}$; rs 12225799, chr11:g.241124C $>\mathrm{G}$ at $11 \mathrm{p} 15$, BET1L/RIC8A, $\quad P=1.1 \times 10^{-21} ; \quad$ rs17332320, $\quad$ chr22: g. $40711620 \mathrm{G}>\mathrm{T}$ at $22 \mathrm{q} 31$, TNRC6B, $P=1.6 \times 10^{-12}$; all $P$-values are of BOLT-LMM results). When the same variants were included in our results, the direction of effect of previously reported variants were concordant. For OC, we identified two loci (rs79219469, chr16:g.54587853 C > T at 16q12, LINC02183, $P=3.3 \times 10^{-8}$; rs567534295, chr17: g. $41200107 \mathrm{C}>\mathrm{T}$ at $17 \mathrm{q} 21, B R C A 1, P=3.1 \times 10^{-8}$; all $P$ values are of BOLT-LMM results). Of the previously reported variants, most have directionally concordant results. However, rs58722170, chr1:g.38096421 G $>\mathrm{C}$ at 1p34, RSPO1, rs2165109, chr2:g.111818658 A > C at 2q13,
ACOXL, rs7953249, chr12:g.121403724 G > A at 12q24, HNF1A-AS1, and rs183211, chr17:g.44788310 G > A at $17 \mathrm{q} 21, N S F$, reported for its association with high-grade serous OC, and rs752590, chr2:g.113972945 A > G at 2q14, PAX8-AS1, rs112071820, chr3:g.138849113_138849114 insGATTCAGAATCCA at 3q23, MRPS22, and rs688187, chr19:g.39732752 G > A at 19q13, IFNL3, reported for its association with mucinous OC, had directionally opposite effect in our samples [7]. Because we have not stratified the cases by the histopathological subtypes, difference in proportions of subtypes may explain this discordance. For UCC, we identified three loci (rs140668832, chr6: g. $30479914 \mathrm{~A}>\mathrm{T}$ at $6 \mathrm{p} 22$, the $\mathrm{MHC}$ region, $P=2.9 \times$ $10^{-10}$; rs150806792, chr11:g.2179342 C > T at 11p15, INSIGF2, $P=4.9 \times 10^{-8}$; rs140991990, chr17:g.70097851 A > $\mathrm{G}$ at $17 \mathrm{q} 24, \operatorname{SOX} 9, P=3.3 \times 10^{-8}$; all $P$-values are of BOLT-LMM results). For all the previously reported variants, the direction of effect was concordant. Out of these detected loci, two (rs79219469: $\mathrm{C}>\mathrm{T}$ at 16q12, and rs567534295:C $>\mathrm{T}$ at 17q21) for OC [7], and two (rs150806792:C > T at 11p15, and rs140991990:A > G at 17q24) for UCC $[9,10]$ are novel associations in the context of GWAS [2-10]. The variant rs79219469:C > T may affect expression status of several genes by altering Gfil and Irf motifs, of which, Gfil is reported to have functions in oncogenesis. The variants rs150806792: $\mathrm{C}>\mathrm{T}$ and rs 140991990:A > G are reported as enhancer and promoter histone marks in several tissues which are not relevant to uterine cervix, however, alterations in Ets and GATA motifs, for example, may result in transcriptional activity of nearby genes. (Supplementary Table 12). For all associations, analysis under the linear mixed model using BOLT-LMM revealed more significant associations than the usual logistic regression model using mach2dat (e.g., rs 7412010:G $>\mathrm{C}$ at $1 \mathrm{p} 36$ associated with UF showed $P$ values of $2.0 \times 10^{-11}$ in mach2dat and $1.2 \times 10^{-12}$ in BOLT-LMM). Generally, BOLT-LMM showed more significant associations than incorporating correlations among 


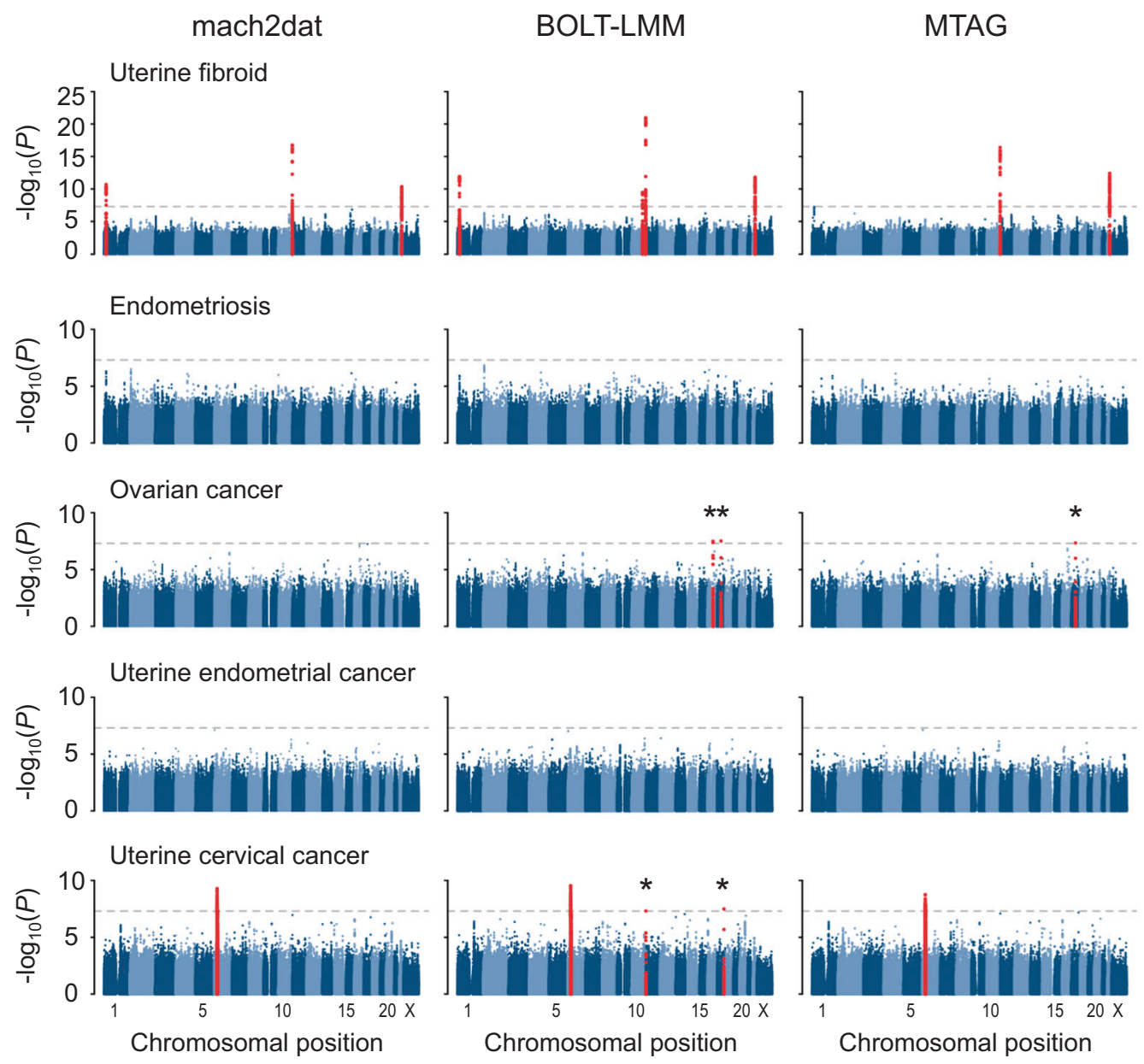

Fig. 1 Manhattan plots of the five GWASs of gynecologic diseases. Manhattan plots of the GWAS of the five gynecologic diseases among Japanese. The $y$-axis indicates $-\log _{10}(P)$ of association of each variant calculated by three methods, including the logistic regression model using mach2dat, the linear mixed model using BOLT-LMM, and incorporating correlations using MTAG, displayed from left to right. Horizontal dashed grey lines indicate genome-wide significance threshold $\left(P<5.0 \times 10^{-8}\right)$. Dots colored in red indicates genome-wide significant loci. Asterisks indicate the novel findings five GWAS estimates using MTAG, however, MTAG showed the most significant association at one locus, rs17332320:G $>\mathrm{T}$ at 22q31. We could not detect significant associations in endometriosis and UEC with any methods. Of the previously reported variants associated with endometriosis, most had the directionally concordant effect except for rs1250241, chr2:g.216 $295312 \mathrm{~T}>\mathrm{A}$ at 2q35, FNl, rs517875, chr3:g.174350886 C $>\mathrm{A}$ at $3 \mathrm{q} 13$, $R A P 1 B P 2$, and rs13271465, chr8:g.17282411 $\mathrm{T}>\mathrm{C}$ at 8p22, MTMR7/ADAM24P. Of the previously reported variants associated with uterine endometrial cancer, most had directionally concordant effect, however, rs1679014, chr9:g.22207037 T >C at 9p21, CDKN2A/CDKN2B, and rs2498796, chr14:g.105243220 G > A at 14q32, AKT1, had directionally discordant or inconsistent effect in our analysis.

There were five associations that reached genome-wide significance by BOLT-LMM but not by mach2dat;
rs12415148:T $>\mathrm{C}$ at 10q24 for UF, rs79219469: $\mathrm{C}>\mathrm{T}$ at $16 \mathrm{q} 12$ and rs567534295:C $>\mathrm{T}$ at $17 \mathrm{q} 21.31$ for $\mathrm{OC}$, and rs150806792:C $>\mathrm{T}$ at $11 \mathrm{p} 15$ and $\mathrm{rs} 140991990: \mathrm{A}>\mathrm{G}$ at $17 \mathrm{q} 24$ for UCC. Of these, four were low-frequency variants $(1 \%<\mathrm{MAF}<5 \%)$. Although some associations were also detected as significant by MTAG, there were no variants that surpassed the genome-wide significance only by MTAG.

\section{Joint analysis of all the cases and controls, random- effect meta-analysis of single disease GWAS, and reverse regression analysis}

We first performed two additional analyses; (i) joint analysis of all the combined cases of the five gynecologic diseases versus shared controls and (ii) random-effect metaanalysis of five single-disease association studies correcting for the overlapping samples using RE2C. 


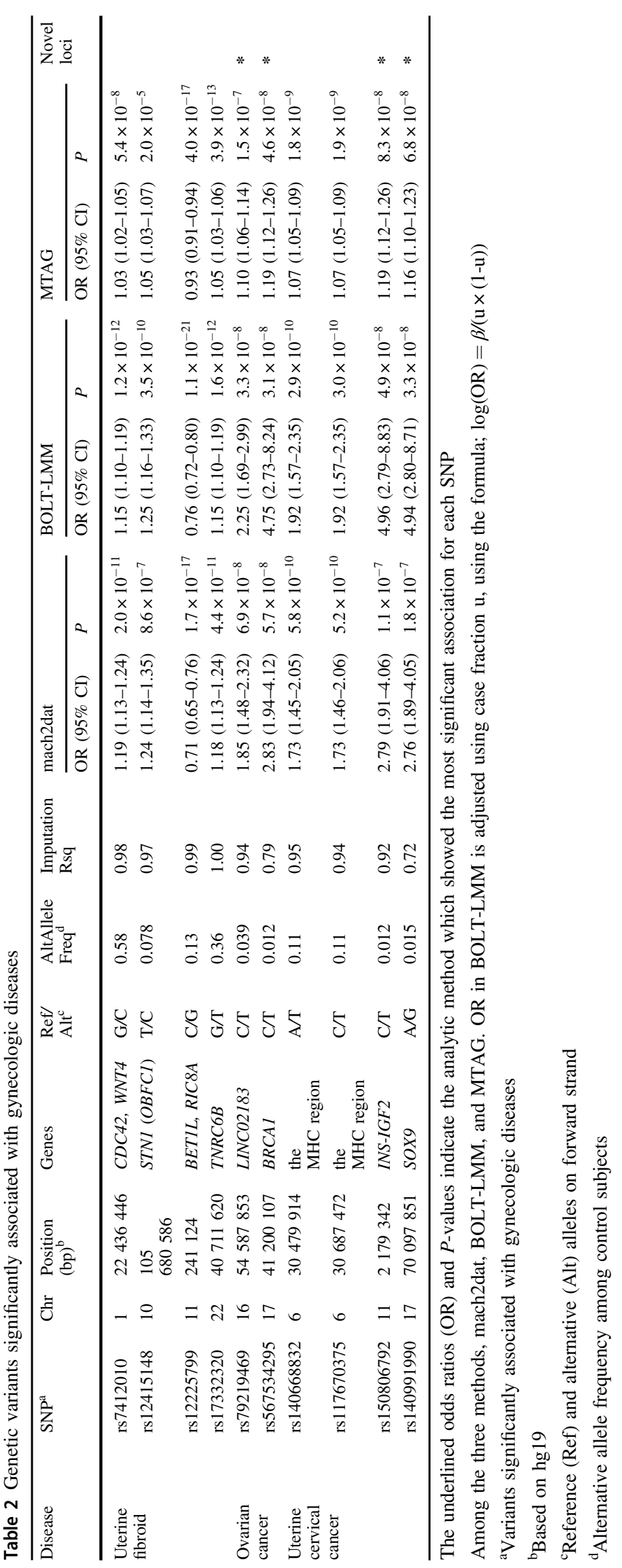


mach2dat

BOLT-LMM

All cases vs controls

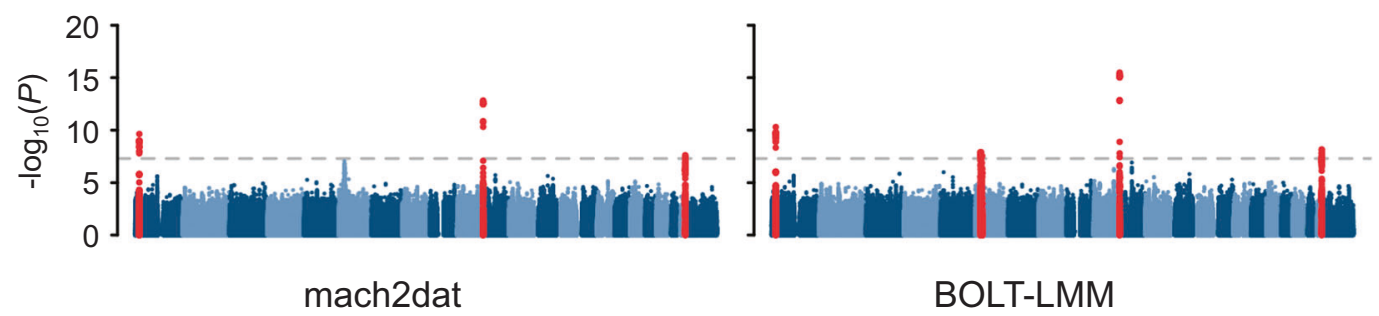

Random effect meta-analysis of the five GWASs of gynecologic diseases (RE2C*)

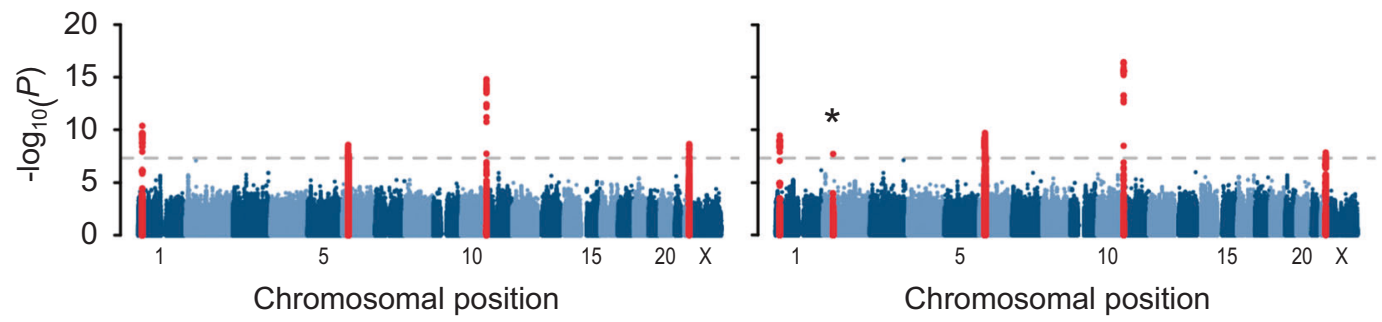

SCOPA

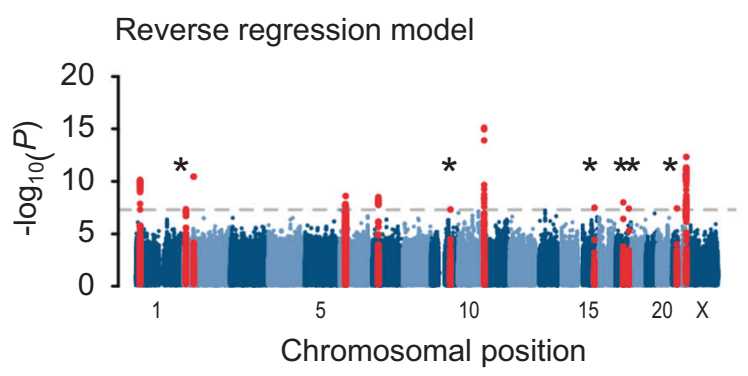

Fig. 2 Manhattan plots of meta-analysis of the five GWASs of gynecologic diseases. Manhattan plots of association $P$-values of all cases versus shared controls (top), cross-trait random effect meta-analysis of the five GWASs of gynecologic diseases controlling for the overlapping samples using RE2C (middle), and the reverse regression analysis using SCOPA (bottom). Analyses under the logistic

Joint analysis of all cases versus shared controls yielded four associations that surpassed the significance threshold (rs7412010:G $>\mathrm{C}$ at $1 \mathrm{p} 36$, rs 12225799: $\mathrm{C}>\mathrm{G}$ at $11 \mathrm{p} 15$, rs $17332320: \mathrm{G}>\mathrm{T}$ at $22 \mathrm{q} 13$, and the $\mathrm{MHC}$ region at 6p22, Fig. 2 and Table 3). Out of the four associations, three (rs7412010:G $>\mathrm{C}$ at $1 \mathrm{p} 36$, rs 12225799: $\mathrm{C}>\mathrm{G}$ at $11 \mathrm{p} 15$, and $\mathrm{rs} 17332320: \mathrm{G}>\mathrm{T}$ at 22q13) were identical to those detected in single disease analysis of UF, and were detected both by mach2dat and BOLT-LMM, while the remaining one, the MHC region, was identical to that found in single disease analysis of UCC, and was significant only in the analysis by BOLT-LMM. For all associations that surpassed the genome-wide significance, BOLT-LMM showed more regression model using mach2dat and under the linear mixed model using BOLT-LMM are displayed from left to right. Horizontal dashed grey lines indicate genome-wide significance threshold $\left(P<5.0 \times 10^{-8}\right)$. Dots colored in red indicates genome-wide significant loci. An asterisk indicates the novel findings

significance than mach2dat, as was shown in the singledisease associations.

Random-effect meta-analysis of the five GWASs of gynecologic diseases using RE2C detected one additional novel association in chromosome 2 (rs937380553, chr2: g. $52063361 \mathrm{~A}>\mathrm{G}$ at $2 \mathrm{p} 16, \operatorname{LOC730100,P}=2.0 \times 10^{-8}$ in RE2C* with BOLT-LMM results, Fig. 2 and Table 3). This detection was achieved only with the use of summary statistics derived from BOLT-LMM. This locus was nominally associated with endometriosis, OC, and UEC $\left(P=2.9 \times 10^{-5}\right.$, $5.5 \times 10^{-4}$, and $1.3 \times 10^{-4}$, in endometriosis, OC, and UEC, respectively, Supplementary Table 13).

We next performed reverse regression analysis using SCOPA. When the MHC region is counted as one locus, we 


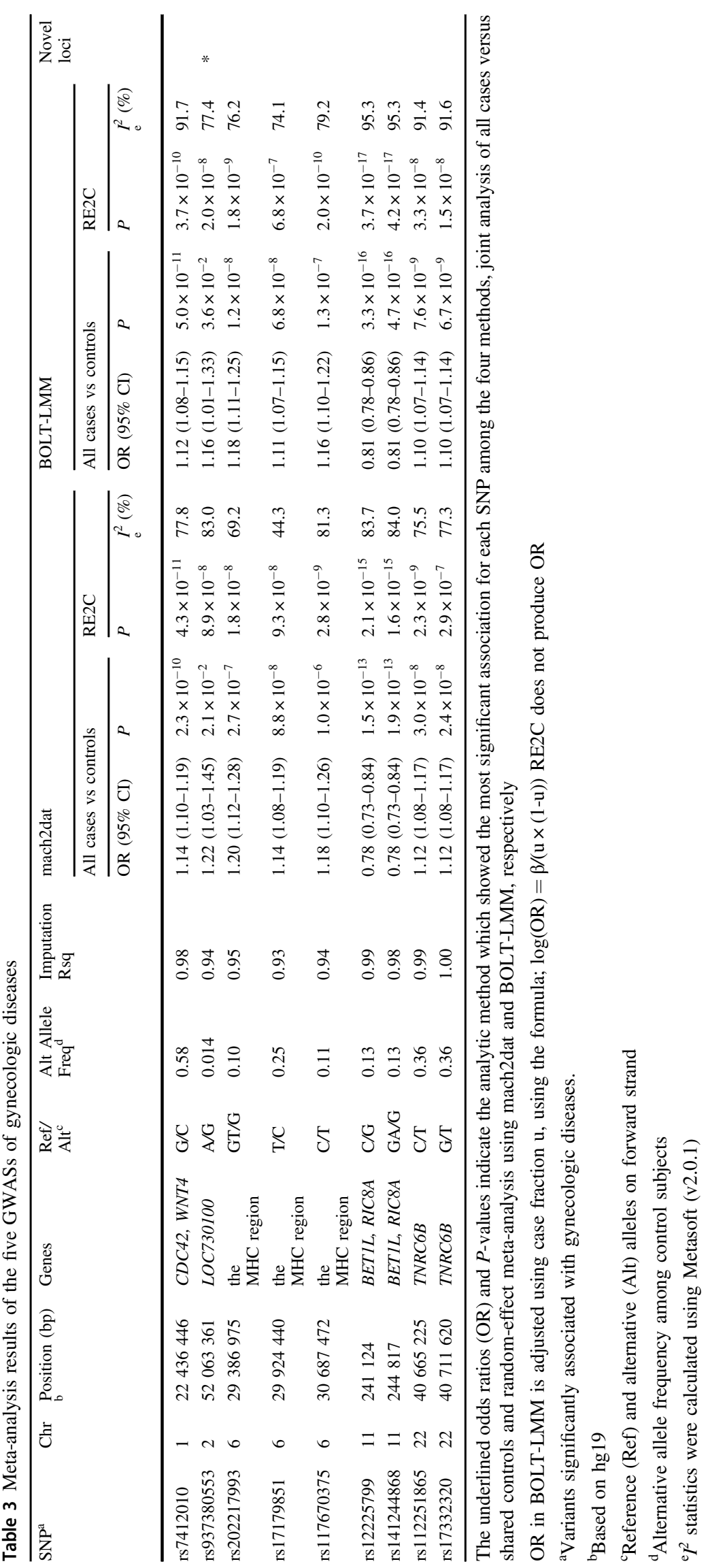


Table 4 Summary statistics of the detected variants in SCOPA

\begin{tabular}{|c|c|c|c|c|c|c|c|c|c|}
\hline $\mathrm{SNP}^{\mathrm{a}}$ & Chr & $\begin{array}{l}\text { Position } \\
\text { (bp) }\end{array}$ & Genes & $\begin{array}{l}\text { Ref / } \\
\text { Alt }^{c}\end{array}$ & AltAlleleFreq $^{\mathrm{d}}$ & Imputation Rsq & $\begin{array}{l}\text { Diseases in the } \\
\text { best model }\end{array}$ & $P$ & Novel loci \\
\hline rs7412010 & 1 & 22436446 & WNT4, CDC42 & $\mathrm{C} / \mathrm{G}$ & 0.42 & 0.98 & UF & $7.0 \mathrm{E}-11$ & \\
\hline rs6432216 & 2 & 11702960 & GREB1 & $\mathrm{T} / \mathrm{C}$ & 0.27 & 1.00 & $\mathrm{UF}+$ Endometriosis & $4.6 \mathrm{E}-08$ & \\
\hline rs937380553 & 2 & 52063361 & LOC730100 & $\mathrm{A} / \mathrm{G}$ & 0.014 & 0.94 & $\begin{array}{l}\text { Endometriosis + OC + } \\
\text { UEC }\end{array}$ & $3.6 \mathrm{E}-11$ & $*$ \\
\hline rs9257985 & 6 & 29652253 & The MHC region & $\mathrm{A} / \mathrm{G}$ & 0.14 & 1.00 & $\mathrm{UF}+\mathrm{UCC}$ & $1.5 \mathrm{E}-08$ & \\
\hline rs117670375 & 6 & 30687472 & The MHC region & $\mathrm{C} / \mathrm{T}$ & 0.11 & 0.95 & UCC & $1.4 \mathrm{E}-08$ & \\
\hline rs2507968 & 6 & 31372718 & The MHC region & $\mathrm{G} / \mathrm{A}$ & 0.74 & 0.95 & $\mathrm{UF}+\mathrm{UCC}$ & $2.5 \mathrm{E}-09$ & \\
\hline rs9271215 & 6 & 32579277 & The MHC region & $\mathrm{C} / \mathrm{T}$ & 0.53 & 0.90 & $\mathrm{UF}+\mathrm{UCC}$ & $2.4 \mathrm{E}-08$ & \\
\hline rs116832992 & 7 & 31784755 & $P D E 1 C$ & $\mathrm{~T} / \mathrm{C}$ & 0.059 & 0.91 & Endometriosis + UCC & $3.2 \mathrm{E}-09$ & \\
\hline rs73494486 & 9 & 101341851 & $G A B B R 2$ & $\mathrm{C} / \mathrm{T}$ & 0.14 & 0.95 & $\mathrm{UF}+\mathrm{OC}$ & $4.8 \mathrm{E}-08$ & $*$ \\
\hline rs138060871 & 11 & 241284 & $B E T 1 L, R I C 8 A$ & G/A & 0.085 & 0.86 & UF & $7.8 \mathrm{E}-16$ & \\
\hline rs145152209 & 15 & 84077212 & $S H 3 G L 3, B N C 1$ & $\mathrm{~A} / \mathrm{G}$ & 0.075 & 0.96 & UF & $3.3 \mathrm{E}-08$ & $*$ \\
\hline $17: 41200107$ & 17 & 41200107 & BRCA1 & $\mathrm{C} / \mathrm{T}$ & 0.012 & 0.79 & $\mathrm{OC}$ & $1.0 \mathrm{E}-08$ & $*$ \\
\hline $17: 70097851$ & 17 & 70097851 & SOX9 & $\mathrm{A} / \mathrm{G}$ & 0.016 & 0.73 & UCC & $3.9 \mathrm{E}-08$ & $*$ \\
\hline rs 147427629 & 21 & 40419321 & LOC107985484 & G/A & 0.023 & 0.84 & $\mathrm{OC}+\mathrm{UEC}$ & $3.8 \mathrm{E}-08$ & $*$ \\
\hline rs 17332320 & 22 & 40711620 & TNRC6B & $\mathrm{G} / \mathrm{T}$ & 0.36 & 1.00 & $\mathrm{UF}+$ Endometriosis & $4.7 \mathrm{E}-13$ & \\
\hline
\end{tabular}

$U F$ Uterine fibroid, $O C$ ovarian cancer, $U E C$ uterine endometrial cancer, $U C C$ uterine cervical cancer

${ }^{a}$ Variants significantly associated with gynecologic diseases

${ }^{b}$ Based on hg19

${ }^{\mathrm{c}}$ Reference (Ref) and alternative (Alt) alleles on forward strand

${ }^{\mathrm{d}}$ Alternative allele frequency among control subjects

${ }^{\text {e}} \mathrm{SCOPA}$ produces $P$-value of the best model

identified 12 loci in association with gynecologic diseases (Fig. 2 and Table 4). Of these, seven loci were best explained when the multiple gynecologic diseases were combined in the regression model. In addition to the three novel loci identified in single disease GWAS and meta-analysis, three loci (rs73494486, chr9:g.101341851 C > T at 9q22, GABBR2, $P=4.8 \times 10^{-8} ; \operatorname{rs} 145152209$, chr15:g.84077212 A $>\mathrm{G}$ at 15q25, SH3GL3/BNCl, $P=3.3 \times 10^{-8}$; and rs147427629, chr21:g.40419321 G $>$ A at 21q22, LOC107985484, $P=$ $3.8 \times 10^{-8}$ ) were novel findings. The top SNPs in the previously identified loci were almost the same as those detected in single disease GWAS and meta-analysis (Tables 2-4). Annotations obtained by searching through HaploReg v4.1 are summarized in Supplementary Table 12.

When comparing the five patterns of the analyses, namely, combination of two conjoining methods (joint analysis of all cases versus shared controls, and randomeffect meta-analysis of the five GWAS correcting for the overlapping subjects), and two analytic methods (the usual logistic model using mach2dat, and the linear mixed model using BOLT-LMM), and reverse regression model, the association results were heterogeneous. These results would suggest that applying multiple methods would offer more opportunity to detect significantly associated loci.

\section{MHC region}

Within those detected loci, the variants showing the most significant associations are almost identical among the analytic methods. However, the MHC region at chromosome 6, which surpassed genome-wide significance in single-disease GWAS of UCC, joint analysis, and randomeffect meta-analysis, showed the most significant associations at different variants among the methods as previously suggested [30]. Fine-mapping and identification of causal variants of the MHC region by high resolution HLA imputation would be warranted $[30,35]$.

\section{BRCA1}

$B R C A 1$ is a tumor suppressor gene well-known from the pedigree studies of familial breast and ovarian cancers. In this study, we identified the significant association with OC at this gene locus in the GWAS approach for the first time. While previous genetic studies mostly focused on coding variants of BRCAl [36], the associated variant, rs567534295:C $>\mathrm{T}$, was the low-frequency noncoding variant $(1 \%<\mathrm{MAF}$ among controls $<5 \%)$, located within the intron between exon 22 and exon 23, and all the variants 
in moderate $\mathrm{LD}\left(r^{2}>0.5\right)$ with rs567534295: $\mathrm{C}>\mathrm{T}$ were not located within the coding region. Rs567534295:C $>\mathrm{T}$ is monomorphic in non-east Asian populations (in 1KGP phase3v5a), suggesting population-specific risk of the variant on OC. To make functional annotation of this lowfrequency risk variant, we assessed the existing expression quantitative trait locus (eQTL) databases (GTEx, HGVD, Ishigaki et al. [37]) and applied artificial intelligence(AI)based prediction algorithm on variant functions (ExPecto [38]). However, known eQTL databases and annotation tools do not contain these variants, and AI-based prediction showed that BRCAl expression was not affected by the variant rs567534295:C $>\mathrm{T}$, suggesting the contribution of undetermined mechanisms to the pathophysiology of OC.

\section{Heritability and genetic correlation}

The disease prevalence used for estimating heritability is shown in Table 1. GCTA-GREML applied to the genotype matrix of each disease produced very stringent results; heritability estimated from genotyped SNPs $\left(h^{2}{ }_{S N P}\right.$ in Table 1$)$ ranged from 0.0260 for ovarian cancer to 0.220 for endometriosis (Table 1). We observed that the $h_{S N P}^{2}$ is about the fifteenth to the two thirds of the disease heritability reported in epidemiological studies $\left(h^{2}\right.$ in Table 1).

Genetic correlations among the five gynecologic diseases under the linear mixed model, calculated directly from the genotyped SNPs, are shown in Fig. 3. All correlations among the four gynecologic diseases except for UCC were

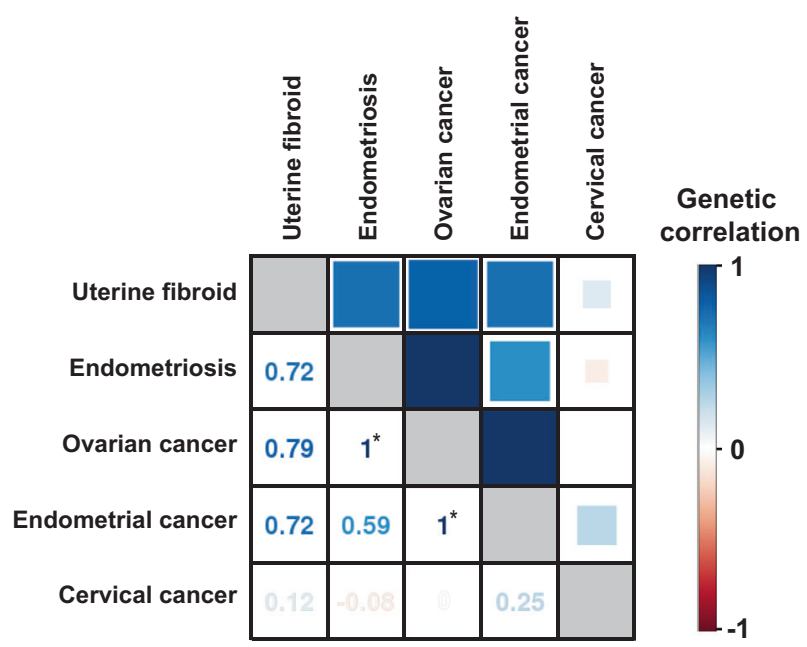

Fig. 3 Cross-trait evaluation of genetic correlation among five gynecologic diseases. Genetic correlations among five gynecologic diseases calculated under the linear mixed model by Haseman-Elston regression using PCGC-s. Correlation is expressed by the color and size of square on the right upper triangle, while represented in digits on the left lower triangle. Asterisks indicate that the real output value exceeded one but was set to one for display purpose directionally positive, and stronger correlations were detected between endometriosis and $\mathrm{OC}\left(r_{\mathrm{g}} \geq 1.00\right)$, OC and UEC $\left(r_{\mathrm{g}} \geq 1.00\right)$, and UF and OC $\left(r_{\mathrm{g}}=0.79\right)$. While relatively weaker and sometimes negative correlations were depicted between UCC and the four other gynecologic diseases $\left(r_{\mathrm{g}}=-0.08-0.25\right.$, Fig. 3$)$.

\section{Discussion}

In this study, we detected nine significant associations with three of the five gynecologic diseases. Four out of the nine associated loci were identified by applying the linear mixed model but not by the usual logistic regression model. For example, rs12415148:T > C at STN1/SLK associated with UF is a known risk locus for UF but was not detected by the usual logistic regression model approach with the current sample size. This supports the application of the linear mixed model approach to the case-control studies to enhance the detection of genetic associations.

We identified two novel loci associated with OC, rs79219469:C $>\mathrm{T}$ and rs567534295:C > T. We compared our results with the publicly available summary statistics from the largest European OC GWAS. We found that chr16 variant rs79219469:C $>\mathrm{T}$ exists in the summary but its effect allele $(T)$ frequency is only 0.0046 , with its association $p$-value 0.84 . We also looked for the chr17 variant rs 567534295:C > T, however, this does not exist in the available summary (monomorphic in 1KG Europeans). We considered that disease risk of these variants were rather population-specific in Japanese (and east Asians).

We identified two novel loci associated with UCC, rs150806792:C > T and rs140991990:A > G. Rs150806792: $\mathrm{C}>\mathrm{T}$ at INS-IGF2 locus is suggested to be associated in the pathogenesis of UCC through the activation of insulin-like growth factor pathway, as reported in the cases of colorectal tumors [39]. Rs140991990:A > G is located at SOX9 locus, which is a member of SOX family and its family member SOX14 is reported to be involved in p53 signaling pathway in a UCC cell line [40]. Also, inhibition of SOX9 is reported to increase radiosensitivity in gastrointestinal cancer [41]. These suggest the roll of SOX9 in the pathogenesis of UCC.

Of the nine identified associations, three top variants; rs567534295:C $>\mathrm{T}$ for OC, and rs150806792: $\mathrm{C}>\mathrm{T}$ and rs140991990:A $>$ G for UCC, are variants found only in Japanese and/or East Asians with rare to low-frequency but not observed in other populations, according to 1KGP phase $3 \mathrm{v} 5 \mathrm{a}$ data. These three were also the variants better detected under the mixed model. Of note, the well-known BRCAl locus, encompassing the low frequency noncoding variant of rs567534295:C > T, was shown to be significantly associated with $\mathrm{OC}$ for the first time in the context of 
GWAS [7]. We note that risk identification of rs567534295: $\mathrm{C}>\mathrm{T}$ could have been achieved only by using the large population-specific imputation reference panel [25, 26], with adequate GWAS sample size, and by using ingenious analytical methods. While the GARFIELD analysis suggested functional annotations of genetics of the phenotypes, cell specificity observed in the results were still relatively nonspecific. Further approaches would be warranted to further elucidate undetermined disease mechanisms.

In the joint analysis, since sample sizes are different among the diseases, signals are most likely to be driven by the disease with the largest samples. As expected, three signals were concordant with those detected in UF GWAS, having the largest samples among the five GWASs, however, signals in the $\mathrm{MHC}$ region were also significant under the linear mixed model, which were concordant with those detected in UCC GWAS, having the smallest samples. This showed that strength of the signals in joint analysis can be either amplified or attenuated depending on the sample sizes and correlations among the diseases at the signal of the interest.

A novel association was also discovered by random-effect meta-analysis of the five GWASs adjusting for the overlapping samples. The top associated SNP rs937380553:A > G at 2p16 locus is located within noncoding LOC730100 gene. The function of this IncRNA is not well investigated and further studies are required to elucidate its contribution to the pathogenesis of gynecologic diseases, especially, possibly shared effect among endometriosis, OC, and UEC. We also applied a novel approach, MTAG, which incorporates correlations among multiple GWAS estimates to enhance detection, to gynecologic diseases. In some, but not all, MTAG results were the most significant among those of the usual logistic regression model and the linear mixed model. Therefore, by applying multiple analytic methods, we can have more opportunity to identify novel associations.

In the reverse regression analysis, we identified three additional novel associations. Rs73494486:C $>\mathrm{T}$ at $G A B B R 2$ locus is associated with combined phenotypes of UF and OC. This locus has neither been reported in the GWAS of UF or OC, however, GABBR2 is suggested to have an important role in EGFR signaling through the ERK1/2 pathway, as reported in lung adenocarcinoma [42]. Rs145152209:A > G at SH3GL3/BNC1 locus is associated with UF. SH3GL3 and BNC1 are both neither reported in the context of UF, however, SH3GL3 is reported as a colorectal cancer-associated gene [43], and BNCl is reported to have association with pancreatic cancer [44, 45]. These suggest that this locus may play an role in proliferative property of UF in some undetermined mechanisms. Rs 147427629:G > A at LOC107985484 locus is associated with OC + UEC. This noncoding RNA is not studied well, however, the similarity of OC and UEC supports the mutual pathophysiology conferred by this non-coding RNA.
We showed the genetic correlations under the mixed model among the five gynecologic diseases. As expected from the past genetic and epidemiologic studies [21-23], these correlations were mostly directionally positive. The correlation was stronger between OC and UEC, and between endometriosis and OC, compared with those between UCC and the other four gynecologic diseases, which was concordant with the epidemiological findings [1, 20-22]. Thinking of the pathophysiology, where UCC is mainly caused by infection of human papilloma viruses, which is distinct from other gynecologic diseases, and where some histological subtypes of OC very often co-occur with endometriosis [1], this result would be considered rational. Although genome-wide additive effects are correlated given the strong genetic correlation and similarity of SNP effect sizes among these gynecologic diseases, we could detect only one additional shared locus in the cross-trait meta-analysis. This was because many diseases have polygenic nature and most of the variants have too small effect sizes to be detected. By increasing sample sizes, more shared loci with small effects are expected to be detected. This encourages cross-trait meta-analysis to support improved power to detect shared loci as described above.

Strength of our study includes the followings: First, we conducted the GWASs with the largest sample sizes among Japanese population, which facilitated the detection of novel associations. Second, we performed association analyses under the linear mixed model and also applied MTAG. We demonstrated that application of the mixed model and/or integrating correlations among multiple diseases increased the power of detection. Third, we used the population-specific imputation reference panel, which contributed to the increased number of variants with higher accuracy, especially those specific to the Japanese population such as the risk noncoding variant at BRCA1. Fourth, we demonstrated that combining the GWASs of multiple diseases in a random-effect metaanalysis revealed a novel candidate association. Fifth, we revealed genetic correlations among multiple gynecologic diseases. Shared genetic etiology encourages the investigation of common pathophysiology of the related diseases.

Although our study is the largest GWAS in the Japanese population, our limitation includes the lack of replication study. This is the task for our future study.

In conclusion, we successfully identified nine significant genetic associations with three gynecologic diseases including four novel ones, by applying association analysis under the mixed model and incorporating correlations among multiple GWAS estimates. Further, cross-trait meta-analysis identified five loci including one novel association which is suggested to be a shared risk locus. We also disclosed genetic correlations among multiple gynecologic diseases. We propose to apply new methodologies to increase detection power, and crosstrait analysis to assess shared risks. 


\section{URLs}

OMIM, http://www.omim.org/

EIGENSOFT v6.1.4, https://www.hsph.harvard.edu/a lkes-price/software/

PLINK 1.9b3.3, https://www.cog-genomics.org/plink2

Minimac3, https://genome.sph.umich.edu/wiki/Minimac

1000 Genome Project, http://www.internationalgenome. org/

Eagle, https://data.broadinstitute.org/alkesgroup/Eagle/

mach2dat, https://genome.sph.umich.edu/wiki/Mach2da

$\mathrm{t}$ :_Association_with_MACH_output

Metasoft, http://genetics.cs.ucla.edu/meta/

GWAS catalog, https://www.ebi.ac.uk/gwas/

HaploReg v4.1, https://pubs.broadinstitute.org/mamma 1s/haploreg/haploreg.php

GENCODE v19 (GRCh37.p13), https://www.gencodegenes. org/releases/19.html

GTEx, https://gtexportal.org/home/

HGVD, http://www.hgvd.genome.med.kyoto-u.ac.jp/

ExPecto, https://github.com/FunctionLab/ExPecto

Acknowledgements We would like to thank the participants and researchers from the project who significantly contributed or collected data. We thank K. Yoshino, E. Kobayashi, S. Matsuzaki, and A. Morimoto for their clinical advice. BBJ was supported by the TailorMade Medical Treatment Program (the BioBank Japan Project) of the Ministry of Education, Culture, Sports, Science, and Technology (MEXT) and the Japan Agency for Medical Research and Development (AMED). This study was supported by Bioinformatics Initiative of Osaka University Graduate School of Medicine. Y.O. was supported by the Japan Society for the Promotion of Science (JSPS) KAKENHI (15H05911, 19H01021), AMED (19gm6010001h0004, 19ek0410041h0003, and 19ek0109413h0001), Takeda Science Foundation, Bioinformatics Initiative of Osaka University Graduate School of Medicine, and Integrated Frontier Research for Medical Science Division, Institute for Open and Transdisciplinary Research Initiatives, Osaka University.

Author contributions TM, YU, KT, YK, and YO designed the study. $\mathrm{TM}, \mathrm{SKL}$, and MA performed statistical analyses. MH, KM, YM, and MK collected and managed the samples. MK conducted genotyping. TM and YO wrote the manuscript. All authors helped interpret the data, reviewed, and edited the final manuscript, and approved the submission.

\section{Compliance with ethical standards}

Conflict of interest The authors declare that they have no conflict of interest.

Ethical approval This study was approved by the ethical committee of Osaka University Graduate School of Medicine.

Informed consent All the subjects provided written informed consent as approved by the ethical committee of RIKEN Yokohama Institute and the Institute of Medical Science, the University of Tokyo.
Publisher's note Springer Nature remains neutral with regard to jurisdictional claims in published maps and institutional affiliations.

Open Access This article is licensed under a Creative Commons Attribution 4.0 International License, which permits use, sharing, adaptation, distribution and reproduction in any medium or format, as long as you give appropriate credit to the original author(s) and the source, provide a link to the Creative Commons license, and indicate if changes were made. The images or other third party material in this article are included in the article's Creative Commons license, unless indicated otherwise in a credit line to the material. If material is not included in the article's Creative Commons license and your intended use is not permitted by statutory regulation or exceeds the permitted use, you will need to obtain permission directly from the copyright holder. To view a copy of this license, visit http://creativecommons. org/licenses/by/4.0/.

\section{References}

1. Nagai K, Hayashi K, Yasui T, Katanoda K, Iso H, Kiyohara Y, et al. Disease history and risk of comorbidity in women's life course: a comprehensive analysis of the Japan Nurses' Health Study baseline survey. BMJ Open. 2015;5:e006360.

2. Cha P-C, Takahashi A, Hosono N, Low SK, Kamatani N, Kubo $\mathrm{M}$, et al. A genome-wide association study identifies three loci associated with susceptibility to uterine fibroids. Nat Genet. 2011;43:447-50.

3. Rafnar T, Gunnarsson B, Stefansson OA, Sulem P, Ingason A, Frigge ML, et al. Variants associating with uterine leiomyoma highlight genetic background shared by various cancers and hormone-related traits. Nat Commun. 2018;9:3636.

4. Uno S, Zembutsu H, Hirasawa, Takahashi A, Kubo M, Akahane TA, et al. A genome-wide association study identifies genetic variants in the CDKN2BAS locus associated with endometriosis in Japanese. Nat Genet. 2010;42:707-10.

5. Sapkota Y, Steinthorsdottir V, Morris AP, Fassbender A, Rahmioglu N, De Vivo I, et al. Meta-analysis identifies five novel loci associated with endometriosis highlighting key genes involved in hormone metabolism. Nat Commun. 2017;8:15539.

6. Uimari O, Rahmioglu N, Nyholt DR, Vincent K, Missmer SA, Becker $\mathrm{C}$, et al. Genome-wide genetic analyses highlight mitogenactivated protein kinase (MAPK) signaling in the pathogenesis of endometriosis. Hum Reprod. 2017;32:780-93.

7. Phelan CM, Kuchenbaecker KB, Tyrer JP, Kar SP, Lawrenson K, Winham SJ, et al. Identification of 12 new susceptibility loci for different histotypes of epithelial ovarian cancer. Nat Genet. 2017;49:680-91.

8. O'Mara TA, Glubb DM, Amant F, Annibali D, Ashton K, Attia J, et al. Identification of nine new susceptibility loci for endometrial cancer. Nat Commun. 2018;9. https://doi.org/10.1038/s41467018-05427-7.

9. Chen D, Enroth S, Liu H, Sun Y, Wang H, Yu M, et al. Pooled analysis of genome-wide association studies of cervical intraepithelial neoplasia 3 (CIN3) identifies a new susceptibility locus. Oncotarget. 2016;7:42216-24.

10. Miura K, Mishima H, Kinoshita A, Hayashida C, Abe S, Tokunaga K, et al. Genome-wide association study of HPVassociated cervical cancer in Japanese women. J Med Virol. 2014;86:1153-8.

11. Arai M, Yokoyama S, Watanabe C, Yoshida R, Kita M, Okawa M, et al. Genetic and clinical characteristics in Japanese hereditary breast and ovarian cancer: first report after establishment of HBOC registration system in Japan. J Hum Genet. 2018;63:447-57. 
12. Michailidou K, Lindström S, Dennis J, Beesley J, Hui S, Kar S, et al. Association analysis identifies 65 new breast cancer risk loci. Nature. 2017;551:92-94.

13. Nagai A, Hirata M, Kamatani Y, Muto K, Matsuda K, Kiyohara Y, et al. Overview of the BioBank Japan project: study design and profile. J Epidemiol. 2017;27:S2-S8.

14. Loh P-R, Tucker G, Bulik-Sullivan BK, Vilhjálmsson BJ, Finucane HK, Salem RM, et al. Efficient Bayesian mixed-model analysis increases association power in large cohorts. Nat Genet. 2015;47:284-90.

15. Turley P, Walters RK, Maghzian O, Okbay A, Lee JJ, Fontana MA, et al. Multi-trait analysis of genome-wide association summary statistics using MTAG. Nat Genet. 2018;50:229-37.

16. Mägi R, Suleimanov YV, Clarke GM, Kaakinen M, Fischer K, Prokopenko I, et al. SCOPA and META-SCOPA: Software for the analysis and aggregation of genome-wide association studies of multiple correlated phenotypes. BMC Bioinform. 2017;18:4-11.

17. Parasar P, Ozcan P, Terry KL. Endometriosis: epidemiology, diagnosis and clinical management. Curr Obstet Gynecol Rep. 2017;6:34-41.

18. Renehan AG, Tyson M, Egger M, Heller RF, Zwahlen M. Bodymass index and incidence of cancer: a systematic review and meta-analysis of prospective observational studies. Lancet (Lond, Engl). 2008;371:569-78.

19. Bhaskaran K, Douglas I, Forbes H, dos-Santos-Silva I, Leon DA, Smeeth L. Body-mass index and risk of 22 specific cancers: a population-based cohort study of $5 \cdot 24$ million UK adults. Lancet (Lond, Engl). 2014;384:755-65.

20. Day FR, Thompson DJ, Helgason H, Chasman DI, Finucane H, Sulem P, et al. Genomic analyses identify hundreds of variants associated with age at menarche and support a role for puberty timing in cancer risk. Nat Genet. 2017;49:834-41.

21. Lu Y, Cuellar-Partida G, Painter JN, Nyholt DR.Australian Ovarian Cancer Study, International Endogene Consortium (IEC) et al. Shared genetics underlying epidemiological association between endometriosis and ovarian cancer. Hum Mol Genet. 2015;24:5955-64.

22. Painter JN, O'Mara TA, Morris AP, Cheng THT, Gorman M, Martin L, et al. Genetic overlap between endometriosis and endometrial cancer: evidence from cross-disease genetic correlation and GWAS meta-analyses. Cancer Med. 2018;7:1978-87.

23. Cuellar-Partida G, Lu Y, Dixon SC, Australian Ovarian Cancer Study, Fasching PA, Hein A, et al. Assessing the genetic architecture of epithelial ovarian cancer histological subtypes. Hum Genet. 2016;135:741-56.

24. Kanai M, Akiyama M, Takahashi A, Matoba N, Momozawa Y, Ikeda $M$, et al. Genetic analysis of quantitative traits in the Japanese population links cell types to complex human diseases. Nat Genet. 2018;50:390-400.

25. Okada Y, Momozawa Y, Sakaue S, Kanai M, Ishigaki K, Akiyama M, et al. Deep whole-genome sequencing reveals recent selection signatures linked to evolution and disease risk of Japanese. Nat Commun. 2018;9:1631.

26. Akiyama M, Ishigaki K, Sakaue S, Momozawa Y, Horikoshi M, Hirata $\mathrm{M}$, et al. Characterizing rare and low-frequency heightassociated variants in the Japanese population. Nat Commun. 2019. (In press).

27. Lee $\mathrm{CH}$, Eskin E, Han B. Increasing the power of meta-analysis of genome-wide association studies to detect heterogeneous effects. Bioinformatics. 2017;33:i379-88.

28. Kanai M, Tanaka T, Okada Y. Empirical estimation of genomewide significance thresholds based on the 1000 Genomes Project data set. J Hum Genet. 2016;61:861-6.
29. Watanabe K, Taskesen E, van Bochoven A, Posthuma D. Functional mapping and annotation of genetic associations with FUMA. Nat Commun. 2017;8:1826.

30. Hirata J, Hosomichi K, Sakaue S, Kanai M, Nakaoka H, Ishigaki $\mathrm{K}$, et al. Genetic and phenotypic landscape of the major histocompatibilty complex region in the Japanese population. Nat Genet. 2019;51. https://doi.org/10.1038/s41588-018-0336-0.

31. Iotchkova V, Ritchie GRS, Geihs M, Morganella S, Min JL, Walter K, et al. GARFIELD classifies disease-relevant genomic features through integration of functional annotations with association signals. Nat Genet. 2019;51:343-53.

32. Weissbrod O, Flint J, Rosset S. Estimating SNP-based heritability and genetic correlation in case-control studies directly and with summary statistics. Am J Hum Genet. 2018;103:89-99.

33. Yasui T, Hayashi K, Nagai K, Mizunuma H, Kubota T, Lee JS, et al. Risk profiles for endometriosis in japanese women: results from a repeated survey of self-reports. J Epidemiol. 2015;25:194-203.

34. Cancer Registry and Statistics. Cancer information service. Japan: National Cancer Center; 2018. https://ganjoho.jp/reg_stat/sta tistics/stat/summary.html. Accessed 1 Oct 2018.

35. Hirata J, Hirota T, Ozeki T, Kanai M, Sudo T, Tanaka T, et al. Variants at HLA-A, HLA-C, and HLA-DQB1 confer risk of psoriasis vulgaris in Japanese. J Invest Dermatol. 2018;138:542-8.

36. Findlay GM, Daza RM, Martin B, Zhang MD, Leith AP, Gasperini M, et al. Accurate functional classification of thousands of BRCA1 variants with saturation genome editing. Nature. 2018;561:294520.

37. Ishigaki K, Kochi Y, Suzuki A, Tsuchida Y, Tsuchiya H, Sumitomo S, et al. Polygenic burdens on cell-specific pathways underlie the risk of rheumatoid arthritis. Nat Genet. 2017;49:1120-5.

38. Zhou J, Theesfeld CL, Yao K, Chen KM, Wong AK, Troyanskaya $\mathrm{OG}$, et al. Deep learning sequence-based ab initio prediction of variant effects on expression and disease risk. Nat Genet. 2018;50:1171-9.

39. Michael PS, Marco HH, Pilar G-C, Norbert S, Andreas W, Stefan F, et al. The IGF1R/INSR inhibitor BI 885578 selectively inhibits growth of IGF2-overexpressing colorectal cancer tumors and potentiates the efficacy of anti-VEGF therapy. Mol Cancer Ther. 2017;16:2223-33.

40. Danijela S, Isidora P, Vladanka V, Marija S, Marija G, Milena S, et al. SOX14 activates the p53 signaling pathway and induces apoptosis in a cervical carcinoma cell line. PLoS ONE. 2017;12: e0184686.

41. Wei Ming-Feng, Kuo Sung-Hsin. Inhibition of SOX9 may be an effective target for increasing radiosensitivity in gastrointestinal cancer. Ann Transl Med. 2017;5:224.

42. Xiaomin N, Fatao L, Yi Z, Zhen Z, Daizhan Z, Ting W, et al. Genome-wide DNA methylation analysis reveals GABBR2 as a novel epigenetic target for EGFR19 deletion lung adenocarcinoma with induction erlotinib treatment. Clin Cancer Res. 2017;23:5003-14.

43. Fang WJ, Zheng Y, Wu LM, Ke QH, Shen H, Yuan Y, et al. Genome-wide analysis of aberrant DNA methylation for identification of potential biomarkers in colorectal cancer patients. Asian Pac J Cancer Prev. 2012;13:1917-21.

44. Li XB, Ma J, Liu ZW, He WF, Li ZZ, Cui M et al. Non-invasive detection of pancreatic cancer by measuring DNA methylation of BNC1 and SEPT9 in plasma. Chin Med J. 2019.

45. Eissa MAL, Lerner L, Abdelfatah E, Shankar N, Canner JK, Hasan NM, et al. Promoter methylation of ADAMTS1 and BNC1 as potential biomarkers for early detection of pancreatic cancer in blood. Clin Epigenetics. 2019;11:59. 